\title{
Les mythes historiques chez Shakespeare : rumeur ou silence?
}

Marie-Madeleine Martinet

\section{(2) OpenEdition}

Journals

Édition électronique

URL : http://journals.openedition.org/shakespeare/490

DOI : $10.4000 /$ shakespeare.490

ISSN : 2271-6424

Éditeur

Société Française Shakespeare

Édition imprimée

Date de publication : 1 novembre 1984

Pagination : 21-30

Référence électronique

Marie-Madeleine Martinet, "Les mythes historiques chez Shakespeare : rumeur ou silence? ", Actes des congrès de la Société française Shakespeare [En ligne], 5 | 1984, mis en ligne le 01 janvier 2007, consulté le 06 mai 2019. URL : http://journals.openedition.org/shakespeare/490 ; DOI : 10.4000/ shakespeare. 490 
SOCIETE FRANÇAISE SHAKESPEARE

Actes du Congrès 1983

\section{MYTHE ET HISTOIRE}

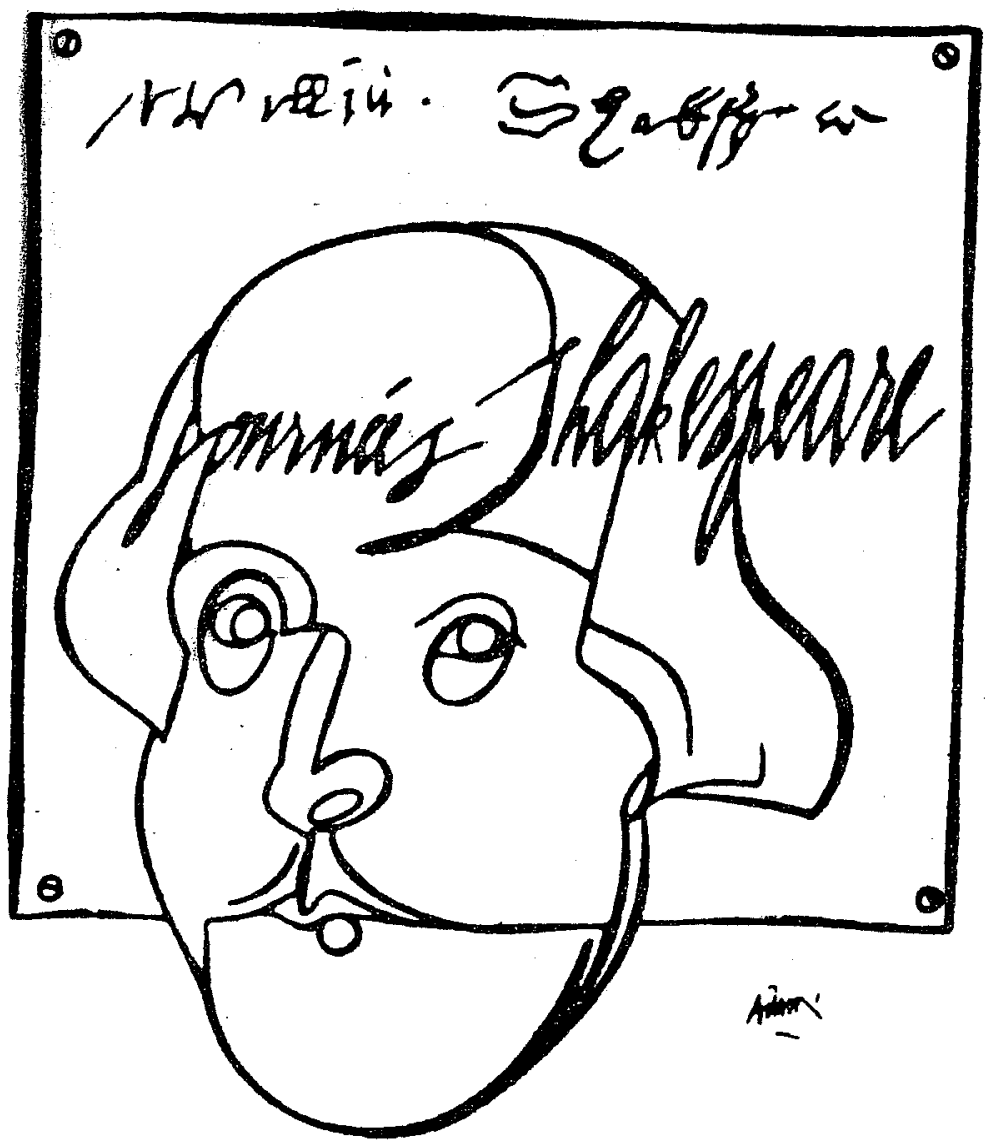

$$
\begin{array}{r}
\text { DiReCteur DE LA' PUblicatic } \\
\text { M.T. Jones-Davie }
\end{array}
$$

Publié avec le concours du Centre National de la Recherche Scientifiq

$$
\text { JEAN TOUZOT Libraire - Editeur }
$$

38, rue Saint-Sulpice 75278 PARIS CEDEX 06199 


\section{LES MYTHES HISTORIQUES CHEZ SHAKESPEARE : RUMEUR OU SILENCE?}

Le genre de la pièce historique a permis à Shakespeare de créer une nouvelle forme de mythe, de mythe qui ferait partie du réel plus qu'il n'en serait l'image, et qui aurait donc du réel toute la richesse de suggestion indéfinie, plus que le sens précis des objets de l'esprit. Les allusions et les points de repère sont comme une rumeur, des références confuses et superposées parce que de nombreux groupes de personnages en sont la source, multipliées du fait qu'elles font aussi appel à un monde extérieur connu du public. La suggestion tient en même temps à l'absence et aux vides pleins de réverbérations, et les silences y sont significatifs ; dans ce monde en partie familier, les manques placés dans une suite d'effets dont ils prennent leur sens sont prolongés par l'imagination; le rythme dispersé est ce qui permet de compléter, et cette esthétique de l'interruption (qui implique liaison) rend perceptible le déroulement du temps qui devient un élément essentiel de la signification ; l'esprit est renvoyé à travers le temps à la fois par le rythme intérieur de la pièce et dans les rappels du passé extérieur : c'est un art de la temporalité adapté à l'histoire. Souvent les rumeurs renvoient aux absences et aux silences. Le fragmentaire est ce qui suscite une unité organique et mouvante.

Les pièces historiques de Shakespeare présentent un paradoxe apparent : les références y sont plus déterminées que partout ailleurs puisqu'elles traitent d'événements et de personnages connus cependant elles sont les meilleurs exemples de mode incertain ou suggestif.

Bardolph et Fluellen sont des noms d'habitants de Stratford cités avec le père de Shakespeare pour n'avoir pas été au culte anglican en $1592^{1}$, mais cette référence éclaire-t-elle les pièces ? Falstaff est un mélange de $\mathrm{Sir}$ John Fastolfe et de Cobham le Lollard - référence assez clairement perçue du temps de Shakespeare pour qu'il 
$\mathrm{y}$ ait eu une défense de Cobham et que le nom d'un personnage de The Merry Wives ait dû être changé ; on retrouve des thèmes connus - les prélats conseillant la guerre, comme au début d'Henry $V$ sont l'un des objets de la critique des Lollards ${ }^{2}$. La plus ancienne copie manuscrite d'une pièce de Shakespeare est précisément celle d'une version abrégée d'Henry $I V$ : le MS de Sir Edward Dering, homme politique et historien ami de Cotton ${ }^{3}$. Mais ces allusions sont ambigües; ces personnages sont, comme leurs sources historiques, des causes d'instabilité ; cependant ils en sont aussi l'opposé et leur image agit par inversion : le protopuritain est devenu un pilier de cabaret ${ }^{4}$; Fastolfe était célèbre pour sa bravoure dans les combats autour de Rouen, et il devint un image légendaire de poltronnerie. Les allusions contribuent à brouiller les références et à leur donner la multiplicité du réel plus qu’à les préciser.

C'est précisément à partir de Falstaff que l'on a d'abord développé la théorie de l'indétermination littéraire, avec Maurice Morgann au XVIII ${ }^{\grave{e}}$ siècle ; dans son Essay on the Dramatic Character of Sir John Falstaff (1777), il dégage l'idée du personnage contradictoire du fait que le dramaturge a réussi à faire percevoir ses traits de caractère à la fois par des «impressions» et par «the understanding»; ce qui crée «an apparent incongruity of character and action» et le rend peu «settled in our minds». Il montre que c'est l'aspect fragmentaire et non terminé de l'insertion dramatique de Falstaff dans la pièce, le fait que les actions dans lesquelles il entre restent incomplètes, qui est source de cet effet ${ }^{5}$.

Pour une pièce historique, la présence de personnages et d'épisodes en partie connus fait que le rapport entre la pièce et le monde extérieur n'est pas de simple imitation et d'image ; plutôt, le monde de la pièce est une partie de l'expérience quotidienne ou héréditaire du public, son aspect fragmentaire s'y prolonge et s'y complète ; il n'y a pas entre la pièce et le réel de rapport terme à terme d'identité, mais de complémentarité. Les allusions ne correspondent pas à l'usage my thique habituel. Leur polysémie n'est pas ordonnée en sens harmoniques, mais en sens 
brouillés comme l'est une rumeur. La multiplicité des références mêle les sens plus qu'elle ne les éclaircit. D'autre part, à l'opposé, les absences de signes sont aussi importantes : les silences, les blancs avec tout l'usage qu'en peut faire la mise en scène, ont un rôle de liaison mystérieuse entre les passages successifs plus que partout ailleurs.

Le glissement de sens tient donc à la fois aux références extrinsèques et à l'organisation intérieure ; une caractéristique des pièces historiques est d'apparaître très différentes à la scène et de prendre leur relief en représentation d'une façon insoupçonnable, à un degré plus marqué que pour toute autre oeuvre : Kenneth Tynan par exemple a noté qu'on ne les découvre qu'en représentation :

I suspected it at Stratford four years ago [1964] and now I am sure; for me the two parts of Henry $I V$ are the twin summits of Shakespeare's achievement, ... great public plays in which a whole nation is under scrutiny and on trial ${ }^{6}$

L'indétermination est causée en partie par la construction indéfinie et dispersée qui contribue à ce double sentiment d'intimité et de mystère. Les variations de rythme suggèrent un glissement perpétuel vers les franges de l'histoire. C'est déjà dans les images du temps, souvent du temps mystérieux et hors du chemin évident, qui peuvent être soulignées dans la mise en scène : les images de lune dans $L a$ première partie d'Henry $I V$, qui sont aussi parfois mises en valeur visuellement. Le nocturne se poursuit dans la deuxième partie de façon plus accentuée : c'est la nuit qu'Henry IV médite sur ses charges (III. i.) ; chez Shakespeare, les rois montés sur le trône à la suite de troubles veillent (Macbeth, le nouveau duc de Milan dans The Tempest) ; les autres qui ont succédé naturellement suivent le rythme de la nature. Le silence nocturne est le moment où les troubles de l'histoire se font entendre en rumeur; le changement de rythme, l'interruption ou trou noir que représente la nuit, est 'ce qui permet la suggestion diffuse ; c'est à propos de cet usage du temps historique que 
Shakespeare a trouvé le rôle de l'interruption significative et suggestive par son contexte. Le quatrième choeur d'Henry $V$, nocturne, est le plus long de tous aussi par un phénomène de rythme de la langue qui donne une impression de diffusion - le nombre de répétitions de mots ou d'adjectifs précédant un nom, causant des échos verbaux qui suggèrent la rumeur nocturne :

Now entertain conjecture of a time

When creeping murmur and the poring dark

Fills the wide vessel of the universe (IV Chorus

où paradoxalement c'est l'obscurité, une absence, qui remplit le monde.

Par ailleurs on sent alterner surtout dans Henry IV le temps hâté (《redeem the time» suggéré par 1 Henry $I V$. I. iii. 178-9) et le temps de la fête.

La puissance de suggestion historique tient surtout à des phénomènes de rythme, rythme verbal et aussi rythme d'éléments non verbaux ou, dans la parole, caractères autres que le sens - ce qui explique que ces oeuvres ne prennent toute leur valeur qu'à la scène ${ }^{7}$. Le rythme de la représentation même est varié quand on change de cadre, et la multiplicité des populations invoquées est suggérée par ces combinaisons de rythmes divers de parole et de jeu. Les scènes avec Mortimer et Glendower (1 Henry IV, III) ont lieu comme dans un autre temps, ce qui est ressenti par le public dans les changements de diction - accent gallois, style rhétorique, et par la scène musicale, ainsi que par l'évocation des esprits nocturnes. Ceci donne l'impression d'un monde aux réverbérations et aux limites indéfinies comme la réalité.

Les scènes successives, ou les groupes de personnages à l'intérieur d'une scène, ont des relations peu rigides, souvent comme une composition de trois quarts, qui suppose des rapports familiers par son imprécision même, alors que les groupes de personnages liés par des rapports géométriques rigoureux expriment d'autant plus de distance of- 
ficielle, soit d'une scène à une autre - grands féodaux puis personnages comiques, différents mais unis par des liens imperceptibles du fait qu'ils appartiennent au passé du public dont ils sont les ancêtres, soit dans une même scène, réunions de personnages dont les rapports incertains peuvent être accentués par la mise en scène dispersée, ces groupes servant comme d'arrière-plan les uns aux autres, intermédiaires entre eux avec le cadre, suggérant l'intimité et la pénétration mutuelle des personnages avec les lieux historiques et familiers de l'action.

Les mouvements contradictoires et erratiques à petite échelle rendent en représentation le sens du temps multiple à grande échelle et de la profondeur du temps, évoquée par la présence de plusieurs générations, elles aussi opposées entre la première et la seconde partie. On a le rythme saccadé de la démarche des vieillards : il s'agit surtout de Northumberland dans la première partie, marquant la puissance des féodaux malgré sa faiblesse, le rythme lent, mais dominateur suggérant la présence à plus grande échelle du rythme menaçant de l'histoire; ce sont surtout les personnages populaires dans la seconde partie, en des scènes hors du temps rythmées par leurs gestes maladroits et leur parole répétitive, qui peuvent encore être accentués visuellement par des accessoires amplifiant les mouvements désordonnés (longue plume d'oie balançant au bas d'un parchemin tenu par une main tremblottante pour Shallow par exemple). Il y a le même décalage pour l'enfance : le petit page porte gauchement un énorme mousquet trop lourd pour lui. De façon générale, la mise en scène insiste souvent sur la superposition des moments, et le lien de l'écoulement du temps avec les personnages : décor de branchages ou acteurs eux-mêmes portant des costumes évoquant la végétation, surtout Falstaff.

La présence du lointain dans l'espace et dans le temps, la superposition incertaine des moments est suggérée par les relations ambiguës du verbal et du non-verbal. Le fait même qu'il y ait dans Henry $I V$ deux parties, dont les liens ne sont pas explicites ${ }^{8}$; provoque ces jeux de renvois confus. Le choeur de La deuxième partie d'Henry: IV est 
significatif ; c'est la Rumeur, couverte de langues, qui est donc un personnage unique mais multiple, et qui est aussi parfois représentée à la scène par un choeur de plusieurs voix - effet inverse de diversité de l'identité. Au début d'une pièce qui reprend la précédente, elle place la Rumeur dans un monde déjà connu, mais en donne une vue indirecte et variée : la parole double l'action d'une manière qui ne correspond pas exactement, créant un effet brouillé du fait de leurs rapports ambigus et de la multiplicité même de la Rumeur.

Les liens entre les paroles et les événements, les personnes et leur pensée, sont souvent comparés à des vases pleins ou vides de sens, symbole des modes de signification dans ces pièces où les connotations ne sont pas des images, représentées par des figures visuelles, mais des sens qui se dégagent d'une réalité riche en mystères non explicites. $\mathrm{La}$ rumeur est d'abord «painted full of tongues», ce qui mêle l'image des langues à celle de la plénitude ; et plus loin elle fait que l'année est supposée pleine d'événements :

And who but Rumour, who but only I, Make fearful musters, and prepar'd defence, Whiles the big year, swoln with some other grief, Is thought with child by the stern tyrant War, And no such matter? Rumour is a pipe Blown by surmises...

(2 Henry IV, Induction, 11-16)

Ce réseau métaphorique s'applique aux personnes; il est présent dans le nom même de Shallow ; Falstaff autour de qui tournent ces images est lui-même «a huge full hogshead» (2 Henry IV, II. iv. 61-2), et dans son éloge du xérès dit qu'il a le cerveau «full of nimble, fiery, and delectable shapes» (2 Henry IV, IV. iii. 98-9). De même à la naissance de Glendower.

The front of Heaven was full of fiery shapes

(I Henry IV, III. i. 35). 
C'est par un usage particulier des contrastes et de l'oscillation entre la parole et l'implicite que Shakespeare a rendu ce sens de familiarité. Si certains personnages évoquaient alors des noms de connaissance, cet effet s'est maintenu sous un autre forme, certaines scènes maintenant connues du public étant attendues à chaque représentation, phénomène souvent remarquable dans les pièces historiques. Un passage célèbre où la parole remplace l'action est le récit de la mort de Falstaff au début d'Henry $V$, or cette scène est de celles qui causent le plus de silence dans l'auditoire. Une raison en est sans doute sa préparation dramatique ; les personnages, Pistol, Nym, l'Hôtesse, Bardolph, le page, entrent en scène après le passage héroïque du départ de l'armée, terminé par des vers rimés, alors que ce morceau est en prose ; il y a un changement d'échelle, et un moment de silence pour l'adaptation, prolongé du fait que les acteurs entrent lentement, pour un épisode de rétrospection, alors que le précédent était tourné vers l'avenir. Ce n'est pas comme auparavant une scène où l'on voit le personnage principal, c'est un épisode où l'on parle de lui ; les personnages ont donc un rôle chorique. Tout ce passage est de renvoi à une réalité lointaine, par le moment de silence suivi par celui où Falstaff, un passé, est évoqué par cette rumeur publique.

Mais ce n'est pas un simple contraste avec le passage précédent; les personnages sont connus puisqu'ils sont apparus deux scènes avant, et cette répétition donne l'impression de retrouver un sens du temps familier derrière le temps historique.

La scène même donne cette impression de mélanges flous ; «Arthur's bosom» pour «Abraham's bosom» évoque par la formule d'origine le Lazare qu'est devenu Falstaff, au lieu du riche attablé qu'il était, et par la formule substituée renvoie Falstaff chez les ancêtres mythiques des Tudors; les suggestions fragmentaires non explicites du passage d'《incarnate» à «carnation» et à «whore of Babylon» font supposer le jeu de mot ordinaire sur «carnal» et «cardinal», en même temps qu'inséré au milieu de ces mots, «rheumatic» (qui, étant donné la prononciation 
$\mathrm{du}$ temps pour «Rome», semblait en être dérivé) nous ramène à Falstaff lui-même auprès ces excursions historiques; l'évocation du Ps. 23 dans «green fields》 doit-elle être précédée de «a table of - » qui ajouterait la table du verset 5 aux pâturages du verset 2, comme dans la leçon du Folio, ou, selon l'émendation de Theobald presque toujours acceptée depuis, «babbled of green fields», qui par une fausse étymologie évoquerait la Tour de Babel, phrase devenue proverbiale (dans Sybil de Disraeli par exemple, un personnage revant de titres de noblesse meurt «babbling of strawberry leaves», ornement des couronnes nobiliaires BK. I, Ch. iii). Les proverbes, la sagesse populaire, rattachent l'épisode au cours du temps, puisque Falstaff est mort à marée descendante. Les phrases répétitives, simplement coordonnées de l'Hôtesse font, comme les allusions mélangées, que tout nous est offert en fragments.

Les pièces historiques ont permis à Shakespeare de créer des mythes qui ne soient pas des images explicatives du réel mais qui en aient la diversité comme s'ils en faisaient partie. Il a ainsi forgé une esthétique de l'indéfini par des multiplicités de sens allant des évocations nombreuses imparfaitement superposées de la rumeur ${ }^{9}$, au vide suggestif du silence. Ces allusions à des mythes ou thèmes connus sont toujours insérées par fragments, qui créent dans la conscience du spectateur une allée et venue entre les fragments et le symbole complet, de même que dans la mémoire cette oscillation tend à causer l'émergence de nouveaux mythes où ces éléments épars s'ordonnent. Un corrélat nécessaire de l'esthétique de l'indéfini étant le rôle du spectateur pour compléter le sens, elle s'est dégagée dans le genre historique où l'on peut compter sur le public pour suppléer des allusions qui faisaient partie de leur propre passé. 
NOTES

1. F.E. Halliday, A Shakespeare Companion (Londres, $1952,1964), s v$. Bardolph.

2. Voir par exemple The true copye of a Prolog wrytten about two $C$. yeres paste by John Wycklife, ed. R. Crowley (Londres, 1550 ), Sig. L iv. $v$.

3. Sur ce MS, voir G. E. Dawson et L. Kennedy-Skipton, Elizabethan Handwriting (Londres, 1968), p. 104. Sur Sir Edward Dering, voir J. P. Rosenblatt, "Sir Edward Dering's Milton», $M P$, $79(1982), 376-385$.

4. Sur les transformations historiques du personnage, voir M. Grivelet, ed. Henry IV (Paris, 1983), p. 17.

5. M. Morgann, An Essay on the Dramatic Character of Sir John Falstaff (Londres, 1777 ), passim.

6. Cité par A. R. Humphreys, "The English History Play», in Shakespeare: Select Bibliographical Guides, ed. S. Wells (Lon. dres, 1973 ), pp. $245-6$.

7. Ces commentaires de $m$ ise en scène sont inspirés principalement par le cycle joué à Stratford par la Royal Shakespeare Company pour son centenaire en 1975, avec Emrys James dans le rôle de Henry IV, Alan Howard dans celui de Hal, Clement Mc Callin dans celui de Northumberland, Terence Wilton comme Mortimer, Yvonne Nicholson comme Lady Mortimer, Griffith Jones comme Owen Glendower, Brewster Mason comme Falstaff, Tim Wilton com. me Bardolph, Maureen Prior en Mistress Quickly, Duncan Kemp ou Ben Wellstood puis Peter Bourke en page, Sydney Bromley en Shallow, Trevor Peacock en Silence, Philip Dunbar en Nym, Richard Moore en Pistol, mise en scène de Terry Hands, décors de Farrah, musique de Guy Woolfenden, éclairages de Stewart Leviton, Cf. S. Beauman, The Royal Shakespeare Company's Production of Henry $V$ for the Centenary Season at the Royal Shakespeare Theatre (Oxford, 1976).

8. Voir de nombreuses études sur les relations entre ces deux oeurres, en particulier G. K. Hunter, "Henry $I V$ and the Elizabethan two-part play», RES, NS, 5 (1954), 236-48, et H, Jenkins, The Structural Problem in Shakespeare's Henry IV (Londres, 1956). $S$ ur la suggestivité du langage dans Henry $I V$, voir M. Doran, «Imagery in Richard IJ and Henry IV»,MLR, 37 (1942),113-22. Sur les pièces historiques, voir J. Wilders, The Lost Garden : A View of Shakespea. re's English and Roman History Plays (Londres, 1978).

9. Comme l'a signalé Monsieur le Doyen Ellrodt dans la discussion, l'un des sens étymologiques de «mythe»est «rumeur». 